\title{
Ende und Zukunft des Reiseberichts: ein Reisebericht ohne Reise?
}

Mit den Tristes Tropiques von Claude Lévi-Strauss sind wir in gewisser Weise an eine Grenze des Reiseberichts gelangt, die zumindest dem traditionellen Bericht von einer Reise gesetzt ist. Vielfältige Faktoren spielen hierbei eine Rolle, denken wir nur an die unterschiedlichsten Arten von Zerstörungen, die von den Reisen unter anderem europäischer Reisender in zum Teil noch unerforschte, ja 'unbesuchte' Gebiete unseres Planeten willentlich, mutwillig oder zumeist absichtslos ausgelöst werden. In derartigen Fällen ist schon, wie uns Claude LéviStrauss denkwürdig vermittelt, der simple 'Besuch' zumeist gleichbedeutend mit der Beeinträchtigung, ja mit der schieren Vernichtung einer Kultur. Wären diese Zerstörungen anders zu verhindern als durch ein glattes Verbot derartiger Reisen in die wenigen noch unberührten Regionen unseres Planeten?

Aber auch die von dem französischen Mythenforscher beklagte leichte Vermarktung und Konsumierbarkeit von Reiseberichten macht uns auf die leichte und passive Konsumierbarkeit der Reisen selber aufmerksam, die im Massentourismus eine ungeheure Dimension angenommen haben. Wir fluten nicht nur Venedig oder Mallorca mit riesigen Wellen von Massentouristen, sondern auch einzelne Inseln der Karibik oder des Stillen Ozeans, die wir mit unseren hochmodernen Kreuzfahrtschiffen aufsuchen. Es gibt kaum noch einen Ort auf unserem Planeten, der vor derartigen Beeinträchtigungen und Zerstörungen sicher wäre. Bedeutet all dies das Ende des Reiseberichts? Ist der Reisebericht also ein Genre, eine Gattung, die an ihr Ende gekommen ist?

In der zweiten Hälfte des 20. Jahrhunderts haben sich die Formen und Normen des literarischen Reiseberichts stark und nachhaltig verändert. Sie reagieren damit auf die Gefahren, die ein Claude Lévi-Strauss bereits in den dreißiger, vierziger und fünfziger Jahren des vergangenen Jahrhunderts klar benannte. So entwickelte der literarische Reisebericht hermeneutische Bewegungsfiguren und Verstehensformen, die nicht mehr einfach konsumierbar sind, sondern ihrem Verstehen und Begreifen klare Hemmnisse, klare Widerständigkeiten in den Weg legen. Sie lassen sich nicht mehr itinerarisch, chronologisch oder themenorientiert lesen, verweigern sich einer autobiographischen Lesart ebenso wie dem Lesemodus eines Tagebuches oder journal intime. Wohin aber entwickelt sich dann der Reisebericht?

Verfolgen wir hier zunächst eine wichtige Spur, die uns zurück ins 18. Jahrhundert führt. Was geschieht, wenn dem Leser des Reiseberichts - wie in Diderots Jacques le fataliste dem Leser des Romans - der Zugang zur Totalität durch

Ә Open Access. (C) 2020 Ottmar Ette, publiziert von De Gruyter. (cc) BY-NC-ND Dieses Werk ist lizenziert unter der Creative Commons Attribution-NonCommericial-NoDerivatives 4.0 Lizenz.

https://doi.org/10.1515/9783110650686-011 
Fragmentierung und radikale Offenheit narrativer Strukturen entzogen wird? In Diderots Jacques begegnen wir einer Figur, die zugleich auch eine Bewegungsfigur ist, aber sich einem Verstehen all der diskontinuierlichen Bewegungen, die von ihr ausgehen, radikal entzieht. Kann ein solch offener, dem Stationenschema des neuzeitlichen Reiseberichts mit seiner Inszenierung reiseliterarischer Orte sich entziehender Text überhaupt noch als Reisebericht angesprochen werden? Stoßen wir hier nicht an die Grenze der Gattung, ja an die Grenze der Lesbarkeit?

Der französische Mythenkritiker und Philosoph Roland Barthes, auf den ersten Blick ein Antipode seines Landsmannes Lévi-Strauss, hat sich vor einem Vierteljahrhundert dieser Frage gestellt. Beschäftigen wir uns an diesem Ende des zweiten Teiles unserer Vorlesung mit seinen Antworten:

\footnotetext{
Was wäre ein Reisebericht, in dem es hieße, man bleibe, ohne doch je angekommen, man reise, ohne doch je aufgebrochen zu sein - wo es, einmal aufgebrochen, niemals hieße, man sei angekommen oder nicht angekommen? Ein solcher Bericht wäre ein Skandal, die Erschöpfung der Lesbarkeit durch Blutverlust. ${ }^{1}$
}

Für den Autor von $S / Z$ ist die Lesbarkeit eines Textes kein Muss, sondern eine überwindbare Größe, deren Überwindung stets neue literarische Formen hervorbringt. Der texte lisible ist der Text, der vom modernen Text verändert, ja zerstört werden muss, um die neuen Formen hervorzutreiben.

Die 'fundamentale Forderung nach dem Lesbaren', so heißt es im selben Abschnitt von $S / Z$, zielt auf die Vollständigkeit, die Fülle und Abgeschlossenheit eines Textes, dessen einzelne Teile funktional miteinander verbunden sein müssen. Das lisible ist in gewisser Weise das Plausible: So auch mit Blick auf den Reisebericht.

Vier Momente eines solchen reiseliterarisch lesbaren Textes hebt Barthes hervor: partir / voyager / arriver / rester. ${ }^{2}$ Dies klingt für einen herkömmlichen, traditionellen Reisebericht plausibel. Es gibt wohl keinen Text, der - ähnlich programmatisch und poetologisch radikal wie Diderots Jacques le fataliste dies für die Gattung des Romans tat - bereits im 18. Jahrhundert experimentelle Formen des Reiseberichts entwickelt hätte. Die Impulse kommen folglich von der Literatur und wirken auf den literarischen Reisebericht maßgeblich ein.

Diese Frage betrifft ganz wesentlich die achte Dimension des Reiseberichts, die sich auf die genrespezifischen Modelle und Traditionen bezieht. Man könnte deshalb von einer poetologischen 'Verspätung' des Reiseberichts gegenüber der Gattung des Romans sprechen, was nicht zuletzt im gattungstheoretischen Ort

1 Barthes, Roland: S/Z. Paris: Seuil 1970, S. 112.

2 Ebda. 
der Reiseliteratur und ihrer traditionellen Anbindung an diktionale Ausdrucksund Lesemuster begründet sein dürfte. Dies wiederum hat mit der eher marginalen theoretischen Bedeutung des Genres der Reiseliteratur zu tun, die es unverkennbar dem Roman überließ, seinen experimentellen Charakter zu erproben und zu untersuchen, wie sich in einem prospektiven Sinne die Vektorizität innerhalb eines Bewegungs-Textes weiter entwickeln und entfalten ließ. Der Rückgriff Diderots auf den Roman und dessen Geschichte griff zielsicher auf den ersten Roman der europäischen Moderne zurück und mit Cervantes’ Don Quijote zugleich auf all jene Traditionslinien, welche diesen mit dem Ritterroman, aber auch mit der Reiseliteratur verbanden.

Doch blieb das poetologische Defizit der Bewegungs-Gattung des Reiseberichts über lange Zeit bestehen. Roland Barthes hat bisweilen in seinen theoretischen Schriften, häufiger aber in seinen eigenen Reiseberichten auf diese poetologische Verspätung reagiert und neue Ausdrucksformen entwickelt. Diese Erkundung der Gattung des Reiseberichts verbunden mit der Entwicklung experimenteller Formen kündigt sich bei ihm schon sehr früh an.

Dabei lässt sich der französische Zeichentheoretiker durchaus als ein herausragender Reiseschriftsteller lesen. Einige Beispiele hierfür mögen genügen. Die Reisefragmente von einer Griechenlandreise, die Barthes 1938 unternahm und 1944 veröffentlichte, die auf Erfahrungen der sechziger Jahre zurückgehenden marokkanischen Skizzen, die postum unter dem Titel Incidents erschienen, und das zeitgleich mit $S / Z$ im Jahre 1970 veröffentlichte Japanbuch L'Empire des signes weisen eine fortschreitende Auflösung traditioneller Grundstrukturen des Reiseberichts auf, die für unsere Vorlesung und die damit verbundene Perspektive mehr als nur interessant sind. Denn sie wirkten buchstäblich wegweisend.

Die experimentellen Elemente seines Reise-schreibens lassen sich kaum übersehen. Während in den Incidents noch Reisebewegungen in verschiedenen Fortbewegungsmitteln erscheinen, sind in L'Empire des signes bis auf wenige, allerdings präzise ausgestreute Spuren die Elemente partir, voyager und arriver fast vollständig getilgt. Dafür dominiert in sehr weitgehendem Maße ein rester, das aber keineswegs nur autobiographisch auszulegen wäre. So verschwindet die materielle Reisebewegung weitgehend aus dem Text, der sich allein noch seinen eigenen Bewegungen anvertraut.

Damit rückt Barthes' Schreiben von einem für ihn wichtigen Bezugsautor ab, Montaigne, der „sein Schreiben dem Wandern oder Spazierengehen“ verglich und „im Unterwegs-Sein schon den Sinn und das Ziel des Reisens“ erblickte. ${ }^{3}$ Den Marokko und Japan gewidmeten Texten liegen zwar in der Realität mehrfache

3 Wuthenow, Ralph-Rainer: Die erfahrene Welt, S. 84. 
Reisen und mehr oder minder kurze Aufenthalte in beiden Ländern zugrunde, so dass in Bezug auf die realen Reiseaktivitäten Barthes' von der Grundfigur des Oszillierens zwischen zwei oder mehreren Ländern und Kulturen gesprochen werden kann; doch weist vor allem der Japan gewidmete literarische Text in seiner Anlage keine Strukturen mehr auf, die dem Leser bereitwillig das Oszillieren zwischen zwei Welten als hermeneutisches Grundschema präsentieren würden. Dies zählt zu den grundlegenden Ent-Täuschungen einer Leserschaft, welche an die gängigen Konventionen einer Reiseliteratur gewöhnt war, deren unvermeidliches Ende Claude Lévi-Strauss konstatiert hatte.

Der Reisebericht entzieht sich hier dem Zwang, einen klaren Anfang und ein klares Ende zu bestimmen, reiseliterarische Orte zu modellieren und in einen wie auch immer gestalteten narrativen Ablauf einzubeziehen. Barthes' Reiseberichte sind weder itinerarisch noch chronologisch angelegt, sie pflegen eine Diskontinuität, welche für den französischen Theoretiker Programm war. Und doch ist - wie der Erfolg von L'Empire des signes zeigt - das Faszinierende des Reiseberichts bei diesem Experiment nicht verloren gegangen, sondern auf eine andere, gänzlich unerwartete Weise präsent. Denn auch durch das Reich der Zeichen führen Wege und von diskontinuierlichen Sprüngen geprägte Bewegungen, die vom Lesepublikum kreativ angeeignet werden können. Im rester konzentriert sich gleichsam eine Vektorizität, die nichts zur Ruhe, nichts zum Stillstand kommen lässt.

Die Reiseberichte des 18. und 19. Jahrhunderts entwickeln ihre Anziehungskraft - wie wir ausführlich gesehen haben - keineswegs allein aus ihrem Bezug zu einer außersprachlichen Wirklichkeit, zu einer wie auch immer bestimmten kulturellen Alterität oder zur historisch verbürgten Figur des Reisenden selbst. Dass es nicht primär der Realitätsbezug ist, der das Publikum begeistert, mag auch durch den derzeitigen Erfolg touristischer Reiseangebote belegt werden, die dem Reisenden nicht nur die Reisewege bestimmter historischer Gestalten (etwa Martin Luthers), sondern auch erfundener Figuren (wie Cervantes' Ritter von der traurigen Gestalt) anbieten. Auch dies führt uns einmal mehr vor Augen: Der Reisende (und der Lesende) eignet sich nicht so sehr einen historischen Reiseweg an als eine hermeneutische Bewegung, welche die (materiellen wie wahrnehmungsspezifischen) Bewegungen beim Lesen des Textes verdoppelt. Daraus entsteht das Glück des Lesenden bei dieser Art von Reisebericht: ein Glück des Begreifens, des Verstehens, ein Glück des Sinns und der Sinne. Dabei kann auch die Reise 'auf den Spuren' einer historischen oder literarischen Figur zu einer Erfahrung des Friktionalen werden, wenn dem Reisenden nicht widerfährt, was auch dem Leser von Reiseberichten widerfahren kann: diese ausschließlich an eine bestimmte (zweifellos hypostasierte) außersprachliche 'Realität' rückzubinden. 
Doch zurück zu Roland Barthes und seinem experimentellen Umgang mit den Formen und Normen des Reise-Schreibens. In seiner experimentellen Autobiographie Roland Barthes von Roland Barthes hatte der Zeichentheoretiker 1975 eher beiläufig darauf aufmerksam gemacht, dass er fünf Jahre zuvor „einen TEXT des LEBENS (Texte de la Vie)“ verfasst habe, in den er „durch das Schreiben über Japan einzutreten“ gesucht habe. ${ }^{4}$ Was aber ist unter einem solchen LebensText aus dem hier für unsere Vorlesung gewählten Zusammenhang zu verstehen?

Roland Barthes hielt mit seinen poetologischen Absichten bei der Umgestaltung des Reiseberichts nicht hinterm Berg. Seinem 1970 erschienenen, Text und Bild ikonotextuell verschränkenden Band Das Reich der Zeichen gab er wie in den siebziger Jahren so oft - eine jener Leseanweisungen mit, die demonstrierten, dass der Autor nicht im entferntesten daran dachte, seine Leser frei und ungeleitet 'darauf loslesen' zu lassen. Es war ihm - wie stets - vielmehr daran gelegen, Poetik und Poetologie seiner Texte deutlich zum Ausdruck zu bringen und die metatextuelle Dimension seiner Schriften zu stärken. So heißt es gleich zu Beginn dieses kunstvoll gestalteten Buches, das zwar auf mehrere Japan-Reisen der sechziger Jahre zurückging, das aber als Japan auf Papier und aus Papier nicht mit Japan verwechselt werden sollte:

Will ich mir ein fiktives Volk ausdenken, so kann ich ihm einen erfundenen Namen geben, kann es erklärtermaßen als einen romanesken Gegenstand behandeln, eine neue Garabagne schaffen, um kein reales Land in meiner Phantasie zu kompromittieren (aber damit kompromittiere ich eben diese Phantasie in den Zeichen der Literatur). Ich kann auch ohne jeden Anspruch, auch nur die geringste Realität darzustellen oder zu analysieren (dies sind die großen Gesten des westlichen Diskurses), irgendwo in der Welt (da unten) eine gewisse Anzahl von Zügen (ein Wort mit graphischer und sprachlicher Bedeutung) aufnehmen und aus diesen Zügen ganz nach meinem Belieben ein System bilden. Und dieses System werde ich nennen: Japan. ${ }^{5}$

Von Beginn an steht nicht die Realität, sondern bestenfalls der Realitätseffekt im Vordergrund. Die 'Verankerung' des traditionellen Reiseberichts in der außersprachlichen Wirklichkeit wird gekappt, um sich ganz auf die Vektorizität der traits, der Züge, konzentrieren zu können. Aber was sind diese geheimnisvollen Züge?

Unübersehbar wird hier von Anfang an ein Warnschild aufgestellt: Dieser Texte de la Vie ist weder mit einer Reise noch mit einer Biographie, sondern

4 Barthes, Roland: Roland Barthes par Roland Barthes. In ders. / Marty, Eric (Hg.): Oeuvres complètes. 3 Bde. Paris. Seuil 1993-1995, Bd. 3, S. 39.

5 Barthes, Roland : Barthes, Roland: L’Empire des signes. In ders/Mary (Hg.), Euvres complètes, Bd. 2, S. 747. 
bestenfalls mit einzelnen disparaten Biographemen in Verbindung $\mathrm{zu}$ bringen, die in einer bestimmten Art und Weise angeordnet wurden. Er ist buchstäblich ein Zeichenreich, das reich an Zeichen und an Zügen ist. Dabei mangelt es keineswegs an autobiographischen Bezügen, die wir in einem Text der Reiseliteratur durchaus erwarten. Die Biographeme oder Splitter eines Lebens werden in der Tat - von persönlichen Erlebnissen über den Abdruck einer Photographie Barthes' in einer japanischen Zeitung bis hin zu Spuren, die uns quer durch die Homosexuellenbars von Tokyo führen - mit einer gewissen Frequenz lustvoll in den Text eingestreut. Wir sind ständig in Bewegung begriffen, doch in einer Bewegung, die letztlich nicht zielgerichtet, sondern hochgradig diskontinuierlich ist. Roland Barthes' Reich der Zeichen situiert sich rigoros jenseits eines den abendländischen Diskurs strukturierenden Gegensatzes zwischen 'Fiktion' und 'Realität' und verweigert sich jeglicher Geste darstellender oder dargestellter Wirklichkeit. Denn Barthes' Japan ist ein Japan, ohne dass sein Japan mit Japan zu verwechseln wäre. Es besitzt die Züge Japans.

Und diese Züge, diese traits, sind die Züge des Lebens: des Lebens eines Landes, des Lebens in einem Land, des Lebens vermittels eines Landes. So bilden 'Leben' und 'Text' keinen Gegensatz: Es ist vielmehr Leben im Text: äußere wie innere Bewegung, Vektorizität. Das Barthes'sche Reich der Zeichen ist darauf angelegt, mit großem Zeichenreichtum Zeichenreiche (auch im graphischen Sinne) aufzuzeichnen, die keinen Gegensatz zwischen Fiktion und Realität, sondern ein Spannungsfeld zwischen Text und Leben entstehen lassen. Denn Literatur - und von ihr ist nicht umsonst im incipit die Rede - ist keine dargestellte Wirklichkeit, sondern die literarische Darstellung gelebter und erlebter Wirklichkeiten, die auf keine wie auch immer konstruierte Realität reduziert werden dürfen. Das Verhältnis von Reisen und Schreiben wird folglich auf die dritte Kategorie des Lebens hin ausgerichtet. Vielleicht zeigt sich für Barthes hier am besten, was er in Kritik und Wahrheit vor allem aus der Position des Kritikers als die „Wahrheit des Schreibens“6 bezeichnete: Denn Literatur ist, weil sie mehr ist, als sie ist. Und dies gilt auch und gerade für die Reiseliteratur mit ihrer eigenen Wahrheit des Schreibens, die unabhängig ist von der dokumentarischen Bezugnahme auf eine außersprachliche Wirklichkeit.

Wie äußert sich diese Poetologie des Reiseschriftstellers Roland Barthes in seinen reiseliterarischen Texten? Inwiefern experimentieren diese mit neuen Formen und Selbstverständnissen des Reiseberichts? Bereits die marokkanischen Incidents (Zwischenfälle) konstruierten nicht nur eine einfach referentialisierbare außersprachliche Realität, sondern vor allem eine Textualität, die

6 Barthes, Roland: Critique et vérité. In ders./Marty (Hg.), Oeuvres complètes, Bd. 2, S. 51. 
ganz im Sinne der Texttheorie rund um Tel Quel auf eine Befreiung des Signifikanten vom Druck der Wirklichkeitsdarstellung, der bloßen Mimesis, abzielte. Hier werden wieder unsere Überlegungen zu Erich Auerbachs Hauptwerk Mimesis fruchtbar, gerade auch mit Blick auf die Darstellungstraditionen einer Totalität von Welt. Denn es ist keineswegs so, dass Barthes den friktionalen Charakter von Reiseliteratur negiert, er deutet ihn nur auf neue Weise und spielt mit der Befreiung des Signifikanten gerade auch in seiner Reiseliteratur. Daher auch die Rede davon, ein eigenes System von 'Zügen', von Vektoren und von Vektorizität, zu konstruieren.

Roland Barthes ging es auch in Das Reich der Zeichen um eine Dezentrierung abendländischer Sinnstrukturen, hinter der nicht mehr nur - wie dies noch am Beispiel des schwarzen Soldaten in den Mythologien der Fall war eine antikolonialistische Haltung, sondern die fundamentale Absicht stand, eine (wie es in einem erstmals 1968 im Nouvel Observateur erschienen Text hieß) „Enteignung des Abendlands“ voranzutreiben, bei der „das abendländische Subjekt nicht mehr Zentrum oder Blickpunkt" ${ }^{\text {"7 }}$ sein konnte und sollte. Die postkoloniale Stoßrichtung ist hier offenkundig - aber sie ist grundlegender Natur und erfasst die Fundamente abendländischen Denkens insgesamt.

Daher der von Beginn an wahrnehmbare Versuch, nicht nur das abendländische Subjekt oder die scheinbare 'Natürlichkeit' des Gesichts, sondern auch den Gegensatz von Schrift und Bild auszublenden. Überhaupt werden von Barthes - zumindest auf den ersten Blick - alle abendländischen Oppositionen hinterfragt, ja in gewisser Weise unschädlich gemacht. Schrift-Bild und BilderSchrift bedingen sich wechselseitig: keineswegs nur in den japanischen SchriftZeichen, sondern auch in der eingeblendeten abendländischen Hand-Schrift, die den Körper gleichsam unter der Hand in den Text holt. Denn die Körperlichkeit ist nicht zuletzt eine Schriftbildlichkeit, die sich jeweils in Schrift wie in Bilder(sequenzen) rückübersetzen lässt.

Doch um was für einen Text handelt es sich bei Barthes' L'Empire des signes? Wir haben schon gesehen, dass er als reiseliterarischer Text das Aufbrechen, Ankommen und Reisen weitestgehend ausblendet und sich diskontinuierlichen Bewegungen, aber auch der Überlappung unterschiedlicher zeichenreicher Sequenzen überlässt, so dass jede Chronologie außer Kraft gesetzt ist. Das von Barthes errichtete Reich der Zeichen, das ebenso den Gegenstand ('Japan') wie zugleich dessen Verfertigung beim Schreiben bezeichnet, ordnet sich überdies weder eindeutig dem Bereich der 'Zeichen der Literatur' noch dem Bereich der Zeichen einer Wissenschaft, einer Analyse außersprachlicher

7 Barthes, Roland: Sollers écrivain. Paris: Seuil, S. 47. 
Wirklichkeit zu. Nicht einmal die von Barthes mitbegründete Semiologie führt hier ihre Analysekategorien ein und macht sie fruchtbar. Zumindest nicht als Wissenschaft.

Aber sehr wohl als Grundlage und Impuls einer écriture, die von der Wissenschaft abstrahiert, sich von ihr förmlich 'abzieht', um sich von ihren Regeln und ihrem Gehäuse zu befreien. L'Empire des signes konstruiert sich vielmehr aus der fundamentalen Diskontinuität einzelner 'Züge' und 'Striche', jener Bildlichkeit von Schriftzügen, wie sie auch und gerade in ihrer Verfertigung und Prozessualität in Das Reich der Zeichen buchstäblich und schriftzüglich eingehen. Dabei ist es keineswegs nebensächlich, dass die Sinnlichkeit des Textes mit einer Erotik des Wissens verknüpft wird. Barthes' Japan wird zu einem Paradies des verstellten Sinnes und der vervielfachten Sinne, die beim Lesen Japans aktiviert und sensibilisiert werden. Die aktive Rolle eines gestaltenden, künstlerischen Lesers wird dabei vorausgesetzt, zumindest aber angeregt. Denn Barthes' Texte stehen zumindest an der Wende der sechziger zu den siebziger Jahren im Zeichen jener Geburt des Lesers, die er am Ende seines berühmten Essays 'Der Tod des Autors' angekündigt hatte: Die Leserin und der Leser werden gleichsam zu Mitreisenden in einem Spiel der Signifikanten und der Referenzen.

Dabei lässt sich Barthes’ écriture noch genauer definieren. Ihre Diskontinuität ist inselhaft, von Insel zu Insel springend: archipelisch. Ein archipelisches Schreiben dominiert - nicht allein, weil Japan ein Archipel ist und das Buch aus Mikrotexten besteht, die einerseits für sich abgeschlossen funktionieren, andererseits aber multirelational vernetzt sind. Die in der Mitte des Bandes (gemeinsam mit einem Zitat von Philippe Sollers, mit dem Barthes seit 1963 freundschaftlich verbunden war) platzierte $\mathrm{Hand}^{8}$ mit dem Pinsel malt Schriftzeichen, die dunkle Züge auf weißer Fläche und damit diskontinuierliche Inseln und Archipele auf und aus Papier entstehen lassen. Sie schwimmen inmitten eines Meeres aus Papier.

Auf diese Weise bilden die Mikrotexte einerseits eine jeweils in sich abgeschlossene, ihren Eigen-Sinn besitzende Insel-Welt, konfigurieren sich zugleich aber zu einer relationalen Inselwelt, deren archipelische und gleichzeitig mobile Strukturierung einen Text entstehen lässt, der in stetiger Bewegung und niemals stillzustellen ist. Diese Gleichzeitigkeit von Geschlossenheit und Offenheit, von scheinbarer Bewegungslosigkeit und Bewegungsfülle, von Territorialität und Relationalität prägt das Reisebuch von Barthes über Japan und verleiht ihm seine besondere Faszinationskraft. L'Empire des signes konnte so zugleich

8 Barthes, Roland: L'Empire des signes, S. 787. 
zum Bestseller und zum Longseller avancieren. Denn der Band hat auch die Hochzeiten poststrukturalistischen Schreibens überlebt.

So ist dieser Insel-Text und InselText über das Japan Roland Barthes' aus ikonotextuellen Text-Inseln hergestellt: ein Japan, aus Papier gemacht, ein Japan, wie es auf dem Papier steht und wie es uns in seinen Strichen und Bewegungen, in seinen 'Zügen' diskontinuierlich, aber zugleich als ein gesammeltes und gesamtes Ensemble entgegentritt. Und dort wird deutlich signalisiert, dass die Grenze zwischen Bild und Schrift eine arbiträre abendländische Setzung ist, die leicht und spielerisch außer Kraft gesetzt werden kann. Gemälde, Haikus und handschriftliche Vermerke durchdringen einander: „Wo beginnt die Schrift? Wo beginnt die Malerei?"“9 Grenzen werden friktioniert, ohne doch gänzlich ignoriert zu werden. Reisen erlaubt leichtere, angeregtere Grenzübertritte und lädt das Schreiben dazu ein, ihm zu folgen und Eigen-Logiken zu entwickeln.

Wenn eben von Territorialität die Rede war, so gilt es, diesen Begriff noch etwas zu 'mobilisieren': Denn der Raum wird beim Reisen durch die Bewegung erzeugt. Die Kategorie des Raumes ist folglich als ein Bewegungsbegriff aufzufassen (vgl. Abb. 91: „Drehen Sie das Bild um: / Nichts mehr, nichts anderes, nichts.“, Shikidai-Korridor, abgebildet in Roland Barthes: L'empire des signes) Nichts in diesem Japan ist stabil - gerade auch die Lebensräume und Lebenswelten nicht. Alles ist drehbar, veränderbar, verstellbar und gerade deshalb nur vektoriell vorstellbar. Die Idee nicht allein des Mobilen, sondern des Mobile nimmt konkrete Text-Gestalt an.

Dies zeigt sich auch und gerade beim Barthes'schen Entwurf des japanischen Hauses, bei dem Ähnlichkeiten mit real existierenden Häusern keineswegs ausgeschlossen, sondern - wie das excipit des Bandes zeigt - beabsichtigt sind:

Bei uns besitzt das Möbelstück eine immobiliäre Berufung, während das Haus in Japan oftmals dekonstruiert und kaum mehr ist als ein mobiliäres Element; im Flur, wie im idealen japanischen Haus, in dem es an Möbeln mangelt (oder wo die Möbel rar gemacht werden), gibt es keinen Ort, der auch nur das geringste Eigentum bezeichnete: weder Sessel noch Bett noch Tisch, von denen aus der Körper sich als Subjekt (oder Herr) eines Raumes konstituieren könnte: Das Zentrum ist verweigert (welch brennende Frustration für den abendländischen Menschen, der überall mit seinem Sessel, seinem Bett versehen, Besitzer eines häuslichen Platzes ist). Der Raum ist nicht zentriert und daher auch umkehrbar: Sie können den Flur von Shikidai umdrehen und nichts wird geschehen, abgesehen von einer folgenlosen Verkehrung von oben und unten, von rechts und links: Der Inhalt wird ohne Rückkehr entlassen: Man kann eintreten, hindurchgehen oder sich direkt auf den Fußboden (oder die Decke, wenn Sie das Bild herumdrehen) setzen, es gibt nichts zu ergreifen. ${ }^{10}$

9 Ebda., S. 759.

10 Ebda, S. 821-824. 
Der Barthes'sche Sprachgestus ist der eines ethnographischen Textes, mit dem uns ein reisender Autor mit den Sitten und Gebräuchen einer anderen Kultur, eines anderen Volkes vertraut machen möchte. Das ist Kernbestand abendländischer Alteritätssicht, eines abendländischen othering. Barthes führt hier durch die Hintertür einen okzidentalen Diskurs wieder ein und ordnet ihm eine Alterisierung des Anderen zu, was zeigt, dass keineswegs alle Alteritäten, alle Oppositionen 'dekonstruiert' und der abendländische Diskurs vollständig entmachtet ist. Aber es handelt sich um einen Diskurs, der nicht nur das Andere in seiner Andersheit und Andersartigkeit beschreibt, sondern die Eigen-Logik des abendländischen Subjekts untergraben, unterlaufen und letztlich ad acta legen will. Eben dies ist der Akt der Barthes‘schen dépossession - lange Jahre vor dem postcolonial criticism.

Damit wird zum einen das Zentrum als jener Fixpunkt verabschiedet, an dem sich für den homme occidental ,die Werte der Zivilisation versammeln und kondensieren“. ${ }^{11}$ Es geht gleichsam um eine 'Entkernung' des abendländischen Diskurses: Barthes versucht, dessen Knackpunkte aufzuspüren und diese frontal anzugehen. Was aber heißt es, ein leeres Zentrum zu entfalten? Barthes hatte dies bereits in seinem Band über den Eiffelturm versucht und die zentrale Markierung der französischen Hauptstadt als einen leeren Turm umschrieben: umgeben von Eisengirlanden, die eine Leere umschließen.

Dem leeren Zentrum in der japanischen Hauptstadt, dem centre ville - centre vide entspricht auch die offene Strukturierung des Buches, in der auch Derrida'sche oder Kristeva'sche Begriffe wie dissémination, déconstruction oder signifiance eigentümlich oszillieren, weil sie aus ihrer theoretischen Verankerung gerissen sind und geradezu frei im Barthes'schen Diskurs schwingen. Wie stets erweist sich der französischen Zeichentheoretiker als ein Sprachendieb, der mit seinem Diebesgut frei zu hantieren versteht und die semantischen Spielräume nutzt, die sich ihm bieten. ${ }^{12}$ Barthes verstellt die Diskurse und pflegt seinen Diskurs der Verstellung. Auch die Grenzen zwischen Narrativ und Theorie werden friktioniert: die Theorie ist überall, überall ist Theorie.

Zum anderen ließe sich diese Problematik sehr wohl mit der sich in den siebziger Jahren akzentuierenden Frage Roland Barthes' nach den Möglichkeiten und Grenzen des Zusammenlebens, der Konvivenz, in Verbindung bringen, die er 1976 und 1977 im Rahmen seiner ersten Vorlesung am Collège de France behandelte. Wir dringen hier zu einem Kernpunkt der Barthes'schen Theorie vor,

11 Barthes, Roland: L'Empire des signes, S. 767.

12 Vgl. hierzu Ette, Ottmar: Der Schriftsteller als Sprachendieb. Versuch über Roland Barthes und die Philosophie. In: Nagl, Ludwig / Silverman, Hugh J. (Hg.): Textualität der Philosophie: Philosophie und Literatur. Wien - München: R. Oldenbourg Verlag 1994, S. 161-189. 
einer Frage, die ihn mit wechselnden Antworten ein Leben lang beschäftigte. Wie können wir zusammenleben?

An diesem textuellen Ende von Barthes' L'Empire des signes (das selbstverständlich wieder auf den Anfang verweist) verschränkt sich in der ikonotextuellen Schreibweise dieses excipit jedoch vor allem die Gerichtetheit des Schrifttextes (von links nach rechts, von oben nach unten) mit der Umkehrbarkeit eines Bildtextes, insofern sich die Photographie eines Hausinnenraums, eines Korridors oder Flurs, in alle Richtungen drehen und in der Tat wie eine zweite Sprache lesen (und erleben) lässt. Zentrale Markierungen von Gegensätzen - wie jenem zwischen 'unten' und ‘oben' - sind außer Kraft gesetzt. Ist es nicht der Leser, der alles auf neue Weise mit Leben erfüllt und lebbar macht?

Kommen wir noch einmal auf die Grundfeste des abendländischen Diskurses mit seinen Oppositionen, mit seinen klaren Gegensätzen, zurück. Das Unterlaufen der Grenzen zwischen links und rechts, oben und unten, innen und außen, Mittelpunkt und Rand, bewegt und unbewegt, wird hier wie in einer mise en abyme - und damit letztlich in einer fraktalen Strukturierung - eingefangen und vorgeführt, gleichsam erlebbar und lebbar, ja in Form des japanischen Hauses bewohnbar gemacht. Man kann hier sehen und sitzen, aber nicht besitzen, schauen, aber nicht überschauen: begreifen, aber nicht ergreifen. Es ist zweifellos der Ort einer Barthes‘schen Utopie, eines gleichsam machtfreien Raumes, wie er ihn in Leçon skizziert hatte. Das Reich der Zeichen ist als Zeichenreich ein Experimentierraum, in dem das Abendländische ausgestellt, aber auch verstellt - und damit aus der Fassung gebracht - wird. Die simpelsten Kriterien des Reiseberichts: Sie gelten nicht mehr, verschwimmen.

Machen wir dies an einem weiteren Beispiel deutlich und ziehen wir dazu den Dekonstruktivismus heran. Das offenkundige Spiel mit dem Derrida'schen Begriff der déconstruction macht deutlich, dass die Referenz eine Reverenz, dass die Realität eine Relationalität ist, in der die Texttheorie die Gestalt eines Landes (wie hier eines Hauses) angenommen hat, ohne doch 'bloße' Texttheorie zu bleiben. Doch auf eigentümliche Weise bleibt dieser Text ein Reisebericht, ein Buch über ein Japan, das wie angekündigt ein Japan aus und auf Papier ist: und ein Japan, das sich unserer Reflexion darbietet.

Die oszillierende Bewegung des literarischen (Reise-) Textes erfasst alle Bereiche des Lebens und Zusammenlebens: Wenn es um Lebensmittel geht, ist sogleich von „dezentrierter Nahrung“13 die Rede. Alles wird von der gezielten dépossession de l'Occident erfasst. Das Reich der Zeichen entfaltet die ästhetische

13 Barthes, Roland: L'Empire des signes, S. 758. 
Kraft einer Theorie, die mehr als Theorie ist (und auch zu sein beansprucht), weil sie sich literarisch ins Leben projiziert, ins Leben einspeist, kurz: eine lebbare Theorie darstellt. Diese Einschreibung ins Leben erfolgt auch - und daran lässt die Bildlegende keinen Zweifel - in das Leben ihres Lesepublikums: „Drehen Sie das Bild herum: nichts mehr, nichts anderes, nichts. "14 Und dennoch hat das Lesepublikum keineswegs nichts in Händen. Ist die Dezentrierung nicht ein höchst didaktischer Lernvorgang?

An die Stelle eines festen Raumes mit seinen Koordinaten tritt die Bewegung, die unaufhörlich zu sein scheint und immer wieder von neuem in andere Bewegungen übergeht. Das auf diese Weise konstruierte, ideale japanische Haus konstituiert (wie der Korridor) nicht etwa einen immobilen, statischen Raum des 'Innen', sondern ist als Bewegungs-Raum, als Vektorenfeld aufgebaut, in welchem sich Bewegungen immer weiter fortpflanzen. Es setzt an die Stelle einer Einbahnstraße, eines sens unique, die Vielgerichtetheit einer Kombinatorik aller Bewegungsrichtungen, ein toutes directions, das mit der archipelischen Schreibweise von Barthes' Zeichenreich vergleichbar ist. Die Räume sind aus Bewegungen gemacht: Erst die Bewegungen, die Reisebewegungen, erzeugen die Räume.

Es handelt sich zugleich um reversible, vektorielle Räume für ein Zusammenleben ohne Besitz, mehr noch: ohne jeden Besitzanspruch, ohne jedes Ergreifen-Wollen des abendländischen Subjekts. Doch im Schreiben kommt zugleich ein Leben-Wollen zum Ausdruck, das auch die Frage nach der Konvivenz stellt. Die Herausforderung des Comment vivre ensemble, der Formen und Normen des Zusammenlebens, ist in Das Reich der Zeichen eingeschrieben. Die Bewegungen des reisenden Ich experimentieren mit den Möglichkeiten eines Zusammenlebens, das mehr ist als ein Reisender auf Besuch: es geht um eine transkulturelle Erfahrung, um ein transkulturelles Erleben, in dessen trans sich die Transformation aller Lebensbezüge bereits einschreibt. Es ist eine zentrifugale Kraft, die den Reisenden in L'Empire des signes beherrscht und antreibt.

Aber wie ist das Buch aufgebaut? Zwischen den Mikrotexten aus der Feder von Roland Barthes sind in hoher ikonotextueller Dichte Photographien, Zeichnungen, Gemälde, Kalligraphien, Postkarten, Zeitungsartikel oder handschriftliche Notizen eingestreut, wobei sich letztere auf Photographien oder Skizzen finden, Schriftzeichen im Prozess ihrer allmählichen Verfertigung entstehen oder gedruckte Kommentare als Legenden an die Seite von Abbildungen treten. Wir stoßen in diesem Band auf die unterschiedlichsten, heterogensten Materialien und Dokumente. Doch sie eint der Bezug zu Japan. Alles wirkt, als wäre es gelebt; alles scheint, als wäre es gefunden - und doch ist es oft erfunden.

14 Ebda. S. 783. 
Beherrschend ist die Vektorizität aller Materialien. Alles wird von zeichenreichen Sinnstrukturen gequert, ohne je auf einen einzigen Sinn reduzierbar zu sein: Stets ergeben sich die unterschiedlichsten Sinn-Richtungen. Im Reich der Zeichen führen die Zeichen ihr Eigen-Leben. Und aus dem Schreiben der Zeichen entsteht ein Leben, das in sich die verschiedensten Formen von Lebenswissen aufgenommen hat und Splitter, einzelne Biographeme homosexuellen wie heterokulturellen Zusammenlebens, bietet. Es ist ein Reich von LebensZeichen, das sich in eine Vielzahl von Lebens-Splittern, von Autobiographemen, aufgelöst hat.

Und was ist nun mit Japan, mit dem Gegenstand der Reisebewegungen? Der des Öfteren geäußerte Vorwurf mangelnder japanologischer Kenntnisse geht vor diesem Hintergrund sicherlich an der Sache vorbei und ins Leere. Dies machte bereits die eingangs zitierte Leseanweisung von Roland Barthes klar. Sie verlegte den Schwerpunkt des Bandes deutlich weg von jeglicher Mimesis, von jeglichem Realitätsversprechen. Zugleich aber darf man feststellen, dass sich die Lehre des Zeichentheoretikers auf das bezieht, was er nicht weiß, um diesen Raum des Nicht-Wissens in einen Experimentierraum zu verwandeln, der die unterschiedlichsten Diskurse über Lebensformen und Lebensnormen auf intensivste Weise miteinander kombiniert. Die friktionale Dimension dieser Art von Reiseliteratur ist offenkundig und fordert ein Lesepublikum, welches sich dieser Tatsache bewusst ist.

Roland Barthes' japanische Lebenslehre macht auch vor dem Körper, vor der Körperlichkeit seiner Japaner nicht halt. Dabei erweist sich einmal mehr Geschichtlichkeit als eine Fokussierung von Gesichtlichkeit: von jener visagéité, welche eine Art Ursprache der Zeichenerzeugung darstellt. ${ }^{15}$ In einem mit 'Das Augenlid' überschriebenen Mikrotext werden Überlegungen weiterentwickelt, in denen die architektonische Struktur des japanischen Hauses - die alle Hierarchien zwischen oben und unten, innen und außen, links und rechts unterläuft auf das lebende Objekt, den Körper 'des’ Japaners, genauer: auf das Auge und dessen 'Rahmung' übertragen werden. Dabei ist die Verknüpfung dieses Transfers mit dem Leben aufschlussreich und soll uns hier noch einmal abschließend beschäftigen:

Das westliche Auge ist einer ganzen Mythologie der Seele, die zentral ist und geheim, unterworfen, deren Feuer aus dem schützenden Hohlraum der Augenhöhlen heraus in ein fleischliches, sinnliches, leidenschaftliches Außen strahlt; doch das japanische Gesicht

15 Vgl. hierzu Deleuze, Gilles / Guattari, Félix: Das Jahr Null - Gesichtlichkeit. In: Bohn, Volker (Hg.): Bildlichkeit. Internationale Beiträge zur Poetik. Frankfurt am Main: Suhrkamp 1990, S. 434. 
kennt keine moralische Hierarchie; es ist gänzlich lebendig, lebhaft (im Widerspruch zur Legende von der östlichen Gelassenheit), weil seine Morphologie nicht 'in der Tiefe' gelesen werden kann, d. h. entlang der Achse einer Innerlichkeit; sein Modell ist nicht die Skulptur, sondern die Schrift: Es ist ein weicher, leichter, dichter Stoff (Seide selbstverständlich), ganz einfach und wie unmittelbar in zwei Zügen kalligraphiert. Das 'Leben' liegt nicht im Licht der Augen, es liegt in dem geheimnislosen Verhältnis zwischen einer Oberfläche und ihren Spalten: in diesem Auseinandertreten, dieser Differenz, dieser Synkope, die, wie man sagt, die Leerform der Lust bilden. ${ }^{16}$

Wir haben es hier mit einer Lektüre des ‘japanischen’ Auges und des Augenlids zu tun: wohlgemerkt aus westlicher Perspektive. Denn die Opposition zwischen 'westlichem' und ‘östlichem' Auge wird keineswegs aufgegeben, sondern zugunsten einer Dekonstruktion der Natürlichkeit des abendländischen Auges genutzt. Das Lesen führt zum Leben und zur Lust. Man könnte in dieser Passage - wie auch in jener des japanischen Hauses - die jeweils unterschiedliche Inszenierungsform des Texte de la Vie, jenes LebensTextes sehen, dessen Gewebe, dessen tissu hier gleichsam das Leben wie das Erleben lustvoll in sich aufgenommen hat. Ansätze zu einer Ästhetik der Lust werden erkennbar, die Barthes wenige Jahre später in Le Plaisir du texte in mikrotextuellen Theoremen eindrucksvoll entfalten sollte. Doch bleiben wir bei Barthes und seinem Band L'Empire des signes.

Die rhetorische Gegenüberstellung von Orient und Okzident, die das gesamte Reich der Zeichen durchzieht, erfüllt auch hier die Funktion, weder einer Fortschreibung des europäischen Orientalismus im Sinne Edward W. Saids ${ }^{17}$ noch einer bloßen Verdrängung derartiger diskursiver Alteritätsmuster anheim zu fallen oder willfährig zu sein. L'Empire des signes lässt sich auf ein Spiel mit orientalistischen Versatzstücken ein, lässt sie als Pastiches eines Pastiche, als Dekonstruktionen einer Dekonstruktion aber ins Leere laufen: in eine Lehre, die das lehrt, was sie nicht weiß, von der man aber nicht sagen könnte, sie wisse nicht, was sie lehrt. Denn die Lust an einer programmatischen Enteignung des Abendlands ist in diesem Barthes‘schen Zeichenreich überall zu spüren: Sie dominiert diesen französischen Reisebericht ohne Reise, diese europäische Reisebeschreibung ohne Ankunft und Aufbruch, vor allem aber ohne einen geordneten Bericht.

Dies bedeutet gerade nicht, dass Das Reich der Zeichen als ideologisierender Befreiungstext lesbar wäre. Dank ihrer polysemen literarischen Schreibweise verkommt Barthes' Theorie niemals zur verfestigten Ideologie - auch nicht jener eines Postkolonialismus avant la lettre. Es geht in der soeben zitierten Passage

16 Barthes, Roland: L'Empire des signes, S. 817.

17 Vgl. das Standardwerk von Said, Edward W.: Orientalism. New York: Vintage Books 1979. 
nicht um die Deskription 'des' japanischen Auges oder Augenlids, um die Mimesis einer wie auch immer gearteten Wirklichkeit, sondern vielmehr um die Inszenierung jenes lebendigen, ja lebhaften LebensTextes, dessen Ziel nicht eine bestimmte außersprachliche Realität, sondern eine mit Leben gesättigte textuelle (wie intertextuelle) Relationalität ist. Überall im Barthes'schen Zeichenreich ist Leben im Netz, ist lustvolles Leben im Text. Überall herrscht die Vieldeutigkeit des Sinnes, der Sinne und der Bewegungs-Richtungen vor. Die Reise ist nicht mehr an eine mehr oder minder kontinuierliche Bewegungsrichtung gefesselt, sondern löst sich in eine Vielzahl von Bewegungen auf, die sich in alle Richtungen verstreuen. Auch dies ist eine Form der Polysemie, der Vieldeutigkeit und Vektorizität.

Nicht der Gegensatz zwischen Realität und Fiktion, sondern das Spannungsfeld (und die wechselseitige Verschränkung) von Vorgefundenem, Erfundenem und Erlebtem (einer Sichtweise, die Barthes als 'System' mit dem Namen Japan ausstattet, einer Schreibweise, die als écriture courte archipelische Züge trägt) stehen im Brennpunkt der Bewegungslinien, die Das Reich der Zeichen zu einem faszinierenden Text des Lesens und Erlebens, aber auch des in vielfacher Hinsicht erlesenen Lebens machen. Wie 'In Griechenland' entwirft Das Reich der Zeichen eine Landschaft der Theorie, wobei es sich hier um eine urbane Insellandschaft handelt, die von keinem Subjekt, von keiner einzelnen Subjektposition aus mehr beherrscht werden kann. Es gibt gute Gründe dafür anzunehmen, dass Roland Barthes den literarischen (und philosophischen) Reisebericht neu erfunden hat.

Noch einmal greifen wir hier auf das Archipelische als Denk- und Schreibmodell zurück. Barthes' LebensText reagiert auf die Last abendländischer Zentrierungen, deren Allgegenwart in Das Reich der Zeichen ständig spürbar ist, mit der List einer offenen archipelischen Schreibweise, um daraus eine stets auf dem Relationalen und der Mobilität beruhende Ästhetik der Lust zu entfalten, deren Grundlegung den französischen Theoretiker während der folgenden Jahre bis zur Veröffentlichung von Die Lust am Text im Jahre 1973 intensiv beschäftigen sollte. Der Entwurf einer derartigen Ästhetik der Lust blieb - anders als bisweilen aus philosophischer Sicht zu hören - keineswegs „folgenlos“, 18 sondern erwies sich als überaus kreativ und produktiv. ${ }^{19}$ Roland Barthes hatte sich auf einen Weg begeben, der die Programmatik seines Essays über den 'Tod

18 Mit diesem einzigen Adjektiv wird Die Lust am Text abgefertigt in Kern, Andrea: Schöne Lust. Eine Theorie der ästhetischen Erfahrung nach Kant. Frankfurt am Main: Suhrkamp 2000, S. 9.

19 Vgl. hierzu meinen bereits angeführten Kommentar in Barthes, Roland / Ette, Ottmar (Hg.): Die Lust am Text. Aus dem Französischen von Ottmar Ette. Kommentar von Ottmar Ette. Berlin: Suhrkamp Verlag 2010, S. 390-407. 
des Autors', aber auch des zweiten Teiles von Kritik und Wahrheit auf verblüffende Weise in einem Text einlöste, der mit allen Konventionen abendländischer Reiseliteratur gezielt brach. Roland Barthes hatte offenkundig das von Claude Lévi-Strauss konstatierte Ende der Reisen zur Kenntnis genommen und an diesem Ende aller Reisen und Reisebeschreibungen der Gattung des Reiseberichts eine neue Wendung gegeben.

Halten wir an dieser Stelle unserer Vorlesung, am Ausklang ihres zweiten Teiles, noch einmal deutlich fest: Der im 18. und 19. Jahrhundert beobachtbare und auch an der Wende vom 20. zum 21. Jahrhundert anhaltende Erfolg der hybriden Gattung des Reiseberichts beruht vor allem auf einer Spatialisierung von Denkstrukturen und Verstehensbewegungen, deren Hermeneutik vom Lesepublikum anhand bestimmter für die Wahrnehmung stilisierter Orte mehr oder minder leicht nachvollzogen werden kann. Lesen ist ein Verstehen, das sich seiner Prozesshaftigkeit, seinem Ablauf als Bewegung, in einer Literatur, die Bewegung räumlich in Szene setzt, besonders eindrucksvoll innewird. Das Genre des Reiseberichts gibt diesen Spatialisierungen von Verstehensbewegungen literarischen Raum - und eben dies tut auch Roland Barthes L'Empire des signes.

Alle Versuche, aus der itinerarischen Struktur traditioneller Formen des Reiseberichts auszubrechen, können als Experimente verstanden werden, die darauf abzielen, die Leserinnen und Leser aus der von Lévi-Strauss beobachteten passiven Rolle, die ihm das simple Nachvollziehen bestimmter dargestellter Erfahrungen konsumträchtig nahelegt, zu befreien. Der Leser soll damit wie die Leserin nicht bloßer Nachvollzieher hermeneutischer Bewegungen sein, sondern zum aktiven Leser werden, dessen Polylog mit dem Text die eigentliche Reisebewegung erzeugt. Eben hier siedelt sich das Barthes'sche Projekt an und genau darum ist es auf dieser Ebene auch von so großer Bedeutung.

Wenn wir die Vektorizität in der Reiseliteratur betrachten, so gilt es, diese nicht allein auf der Seite des Schreibens, auf der Seite der Produktivität zu vermuten und $\mathrm{zu}$ analysieren. Erst durch die Leserinnen und Leser wird Literatur letztlich in Bewegung gesetzt, das heißt in eine Dynamik übersetzt, die vorgängige, im Text verankerte Bewegungen in sich aufnimmt und verändert. Vektorizität ist folglich ebenso sehr eine Sache der Rezeption wie der Produktion von Reiseliteratur. Dies gilt es auch für den dritten und abschließenden Teil unserer Vorlesung im Gedächtnis zu behalten.

Wie also: ein Reisebericht ohne Reise? Ein Reisebericht ohne Aufbruch und Ankunft, ohne Höhepunkt und Rückkunft am Ursprungsort des Reisenden? Es gibt ihn, insofern er weniger auf die nachzuvollziehenden Bewegungen des Verstehens als auf ein polylogisches Verstehen in Bewegung abzielt. Dieses aber ist keinesfalls auf den Bereich der Reiseliteratur beschränkt, sondern setzt 
gerade in jenen literarischen Texten, die vorhandene Grenzziehungen verletzen oder missachten, eine Literatur frei, die durch ihre Bewegungen, durch ihre fundamentale Vektorizität gekennzeichnet ist. Dieser Dynamik eines auf den verschiedensten Ebenen und in unterschiedlichsten Kontexten grenzüberschreitenden Schreibens widmen sich die nachfolgenden Einzeluntersuchungen reiseliterarischer Texte, die kein vollständiges Panorama ergeben, wohl aber exemplarisch und aus historischer Perspektive repräsentativ die Möglichkeiten, Potentialitäten und Potenzen des literarischen Reiseberichts ausloten wollen. Sie sollen in ihrer je eigenen Dynamik eine Welt in Bewegung und die Verfahren eines Schreibens darstellen, das weit davon entfernt ist, an ein Ende gekommen zu sein. 